\title{
SYMMETRY AND CONCENTRATION BEHAVIOR OF GROUND STATE IN AXIALLY SYMMETRIC DOMAINS
}

TSUNG-FANG WU

Received 9 October 2003

We let $\Omega(r)$ be the axially symmetric bounded domains which satisfy some suitable conditions, then the ground-state solutions of the semilinear elliptic equation in $\Omega(r)$ are nonaxially symmetric and concentrative on one side. Furthermore, we prove the necessary and sufficient condition for the symmetry of ground-state solutions.

\section{Introduction}

Let $N \geq 2$ and $2<p<2^{*}$, where $2^{*}=2 N /(N-2)$ for $N \geq 3$ and $2^{*}=\infty$ for $N=2$. Consider the semilinear elliptic equation

$$
\begin{aligned}
-\Delta u+u & =|u|^{p-2} u \quad \text { in } \Omega, \\
u & =0 \quad \text { on } \partial \Omega,
\end{aligned}
$$

where $\Omega$ is a domain in $\mathbb{R}^{N}$. When $\Omega$ is a bounded domain in $\mathbb{R}^{N}$ being convex in the $z_{i}$ direction and symmetric with respect to the hyperplane $\left\{z_{i}=0\right\}$, the famous theorem by Gidas, Ni, and Nirenberg [6] (or see Han and Lin [7]): if $u$ is a positive solution of (1.1) belonging to $C^{2}(\Omega) \cap C(\bar{\Omega})$, then $u$ is axial symmetric in $z_{i}$. However, the axially symmetry of positive solution generally fails if $\Omega$ is not convex in the $z_{i}$ direction. For instance, Dancer [5], Byeon [2, 3], and Jimbo [8] proved that (1.1) in axially symmetric dumbbell-type domain has nonaxially symmetric positive solutions. Wang and $\mathrm{Wu}$ [13] and $\mathrm{Wu}$ [15] showed the same result in a finite strip with hole. In this paper, we want to show that the symmetry and concentration behavior of ground-state solutions in axially symmetric bounded domains $\Omega(r)$ (will be defined later), where the domains $\Omega(r)$ are different from those of Dancer [5], Byeon [2, 3], Jimbo [8], and are extensions of Wang and $\mathrm{Wu}[13]$ and $\mathrm{Wu}$ [15]. The definition of ground-state solution of (1.1) is stated as follows. Consider the energy functionals $a, b$, and $J$ in $H_{0}^{1}(\Omega)$,

$$
a(u)=\int_{\Omega}\left(|\nabla u|^{2}+u^{2}\right), \quad b(u)=\int_{\Omega}|u|^{p}, \quad J(u)=\frac{1}{2} a(u)-\frac{1}{p} b(u) .
$$


It is well known that the solutions of (1.1) are the critical points of the energy functional $J$. Consider the minimax problem

$$
\alpha_{\Gamma}(\Omega)=\inf _{\gamma \in \Gamma(\Omega)} \max _{t \in[0,1]} J(\gamma(t))
$$

where

$$
\Gamma(\Omega)=\left\{\gamma \in C\left([0,1], H_{0}^{1}(\Omega)\right) \mid \gamma(0)=0, \gamma(1)=e\right\}
$$

$J(e)=0$ and $e \neq 0$. We call a non zero critical point $u$ of $J$ in $H_{0}^{1}(\Omega)$ with $J(u)=\alpha_{\Gamma}(\Omega)$ a ground-state solution. It follows easily from the mountain pass theorem of Ambrosetti and Rabinowitz [1] that such a ground-state exists. We remark that the ground-state solutions of (1.1) can also be obtained by the Nehari minimization problem

$$
\alpha_{0}(\Omega)=\inf _{v \in \mathbf{M}_{0}(\Omega)} J(v),
$$

where $\mathbf{M}_{0}(\Omega)=\left\{u \in H_{0}^{1}(\Omega) \backslash\{0\} \mid a(u)=b(u)\right\}$. Note that $\mathbf{M}_{0}(\Omega)$ contains every nonzero solution of (1.1) and $\alpha_{\Gamma}(\Omega)=\alpha_{0}(\Omega)$ (see Willem [14] and Wang [12]).

Now, we consider the following assumptions of an axially symmetric unbounded domain $\Omega$. For the generic point $z=(x, y) \in \mathbb{R}^{N-1} \times \mathbb{R}$,

$(\Omega 1) \Omega$ is a $y$-symmetric (axially symmetric) domain of $\mathbb{R}^{N}$, that is, $(x, y) \in \Omega$ if and only if $(x,-y) \in \Omega$;

$(\Omega 2) \Omega$ is separated by a $y$-symmetric bounded domain $D$, that is, there exist two disjoint subdomains $\Omega_{1}$ and $\Omega_{2}$ of $\Omega$ such that

$$
\begin{gathered}
(x, y) \in \Omega_{2} \text { if and only if }(x,-y) \in \Omega_{1}, \\
\Omega \backslash \bar{D}=\Omega_{1} \cup \Omega_{2} ;
\end{gathered}
$$

( $\Omega 3$ ) equation (1.1) in $\Omega$ does not admit any solution $u \in H_{0}^{1}(\Omega)$ such that $J(u)=$ $\alpha_{0}(\Omega)$.

Now, we give some examples. The infinite strip with hole: $\Omega^{\prime}=\mathbf{A}^{r} \backslash \omega$, where $\mathbf{A}^{r}=$ $B^{N-1}(0 ; r) \times \mathbb{R}$ and $\omega \subset \mathbf{A}^{r}$ is a $y$-symmetric bounded domain, and $\Omega^{\prime \prime}=\left\{(x, y) \in \mathbb{R}^{N-1} \times\right.$ $\left.\left.\mathbb{R}|| x\right|^{2}<|y|+1\right\}$. Clearly, $\Omega^{\prime}$ and $\Omega^{\prime \prime}$ satisfy $(\Omega 1)$ and $(\Omega 2)$. Furthermore, by Lien, Tzeng, and Wang [9, Lemma 2.5], if $\Omega$ is a ball-up domain in $\mathbb{R}^{N}$, then (1.1) in $\Omega$ does not admit any solution $u \in H_{0}^{1}(\Omega)$ such that $J(u)=\alpha_{0}(\Omega)$. Thus, the domain $\Omega^{\prime \prime}$ satisfies $(\Omega 3)$. Moreover, along the same line of the proof of Lien, Tzeng, and Wang [9, Lemma 2.5], we obtain $\alpha_{0}\left(\Omega^{\prime}\right)=\alpha_{0}\left(\mathbf{A}^{r}\right)$. By Lemma 2.8, the domain $\Omega^{\prime}$ satisfies $(\Omega 3)$ (or see Wang [12, Example 2.13 and Proposition 2.14]).

Let $\Omega(r)=\Omega \cap B^{N}(0 ; r)$ be a $y$-symmetric bounded domain and let $\Omega_{t}^{+}=\{(x, y) \in \Omega$ | $y>t\}$ and $\Omega_{t}^{-}=\{(x, y) \in \Omega \mid y<t\}$, then our first main result is the following theorem.

Theorem 1.1. Suppose that $\Omega$ satisfies $(\Omega 1),(\Omega 2)$, and $(\Omega 3)$. Then, for each $\varepsilon>0$ and $l \geq 0$ there exists an $\tilde{r}(\varepsilon, l)>0$ such that for $r>\tilde{r}(\varepsilon, l)$, if $v$ is a ground-state solution of $(1.1)$ in $\Omega(r)$, then either $\int_{\Omega_{l}^{+}}|v|^{p}<\varepsilon$ or $\int_{\Omega_{l}^{-}}|v|^{p}<\varepsilon$. 
Note that, if we take $\varepsilon=(p /(p-2)) \alpha_{0}(\Omega)$ and $l=0$, then there exists an $r_{0}>0$ such that for $r>r_{0}$, every ground-state solution of (1.1) in $\Omega(r)$ is not $y$-symmetric. Then, we have the following result.

Corollary 1.2. Let $\varepsilon=(p /(p-2)) \alpha_{0}(\Omega)$ and $l=0$, then there exists an $r_{0}>0$ such that for $r>r_{0}$, (1.1) in $\Omega(r)$ has at least three positive solutions of which one is $y$-symmetric and the other two are not $y$-symmetric.

By Theorem 1.1, for each $\varepsilon>0$ and $l \geq 0$ there exists an $m_{0} \in \mathbb{N}$ such that for each $m \geq$ $m_{0},(1.1)$ in $\Omega(m)$ has a ground-state solution $v_{m}$ that satisfies $\int_{\Omega_{l}^{+}}\left|v_{m}\right|^{p}<\varepsilon$ or $\int_{\Omega_{-l}^{-}}\left|v_{m}\right|^{p}<$ $\varepsilon$. Then, we have the following results.

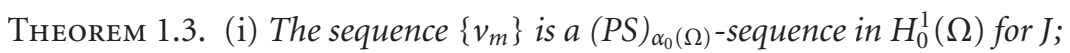

(ii) $v_{m} \rightarrow 0$ weakly in $L^{p}(\Omega)$ and in $H_{0}^{1}(\Omega)$ as $m \rightarrow \infty$.

By Theorem 1.1, the ground-state solutions of (1.1) in $\Omega(r)$ are not $y$-symmetric for large $r$. In this motivation, we consider the positive ground-state solutions of the following equation:

$$
\begin{aligned}
-\Delta u+u & =f(u) \quad \text { in } \Theta, \\
u & =0 \quad \text { on } \partial \Theta,
\end{aligned}
$$

where $\Theta$ is a $y$-symmetric bounded domain and the nonlinear term $f$ is usually assumed to satisfy the following conditions:

( $f 1) f(-t)=-f(t)$ and $f(t)=o(|t|)$ near $t=0$;

( $f 2)$ there exist two constants $\theta \in(0,1 / 2)$ and $C_{0}>0$ such that $0<F(u) \equiv \int_{0}^{u} f(s) d s \leq$ $\theta u f(u)$ for all $u \geq C_{0}$;

(f3) $|f(t)| \leq C|t|^{q}$ for some $1<q<(N+2) /(N-2)$ if $N>2,1<q<\infty$ if $N=2$ and for large $t$

(f4) $\partial^{2} f / \partial t^{2}(t) \geq 0$ for $t \neq 0$.

$f(t)=|t|^{p-2} t$ is a typical example. Under the conditions $(f 1)$ through $(f 3)$, the definition of ground-state solutions of (1.7) is similar to the minimax problem (1.3). Here, we modify the proof of Chern and Lin [4] to get the following results.

Theorem 1.4. Let $v \in C^{2}(\Theta) \cap C(\bar{\Theta})$ be a positive ground-state solutions of (1.7) in $\Theta$. Then, there exists $a z_{0} \in\{y=0\} \cap \Theta$ such that $(\partial v / \partial y)\left(z_{0}\right)=0$ if and only if $v$ is $y$-symmetric.

Corollary 1.5. If $v$ is a positive ground-state solution of $(1.1)$ in $\Omega(r)$ as in Corollary 1.2 and $z_{c}$ is a critical point of $v$, then $z_{c} \notin\{y=0\} \cap \Omega$. In particular, either $(\partial v / \partial y)(z)<0$ or $(\partial v / \partial y)(z)>0$ for all $z \in\{y=0\} \cap \Omega$.

\section{Preliminaries}

We define the $y$-symmetric domains and $y$-symmetric functions as follows.

Definition 2.1. (i) $\Omega$ is $y$-symmetric provided that $z=(x, y) \in \Omega$ if and only if $(x,-y) \in$ $\Omega$;

(ii) let $\Omega$ be a $y$-symmetric domain in $\mathbb{R}^{N}$. A function $u: \Omega \rightarrow \mathbb{R}$ is $y$-symmetric (axially symmetric) if $u(x, y)=u(x,-y)$ for $(x, y) \in \Omega$. 
Throughout this paper, let $\Omega$ be a $y$-symmetric domain in $\mathbb{R}^{N}, H_{s}(\Omega)$ the $H^{1}$ - closure of the space $\left\{u \in C_{0}^{\infty}(\Omega) \mid u\right.$ is $y$-symmetric $\}$ and let $X(\Omega)$ be either the whole space $H_{0}^{1}(\Omega)$ or the $y$-symmetric Sobolev space $H_{s}(\Omega)$. Then, $H_{s}(\Omega)$ is a closed linear subspace of $H_{0}^{1}(\Omega)$. Let $H_{s}^{-1}(\Omega)$ be the dual space of $H_{s}(\Omega)$.

We define the Palais-Smale (PS) sequences, (PS)-values and (PS)-conditions in $X(\Omega)$ for $J$ as follows.

Definition 2.2. We define the following:

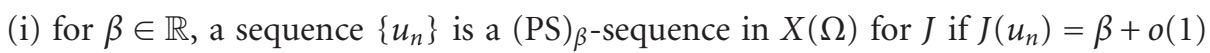
and $J^{\prime}\left(u_{n}\right)=o(1)$ strongly in $X^{-1}(\Omega)$ as $n \rightarrow \infty$;

(ii) $\beta \in \mathbb{R}$ is a (PS)-value in $X(\Omega)$ for $J$ if there is a (PS) ${ }_{\beta}$-sequence in $X(\Omega)$ for $J$;

(iii) $J$ satisfies the (PS $)_{\beta}$-condition in $X(\Omega)$ if every $(\mathrm{PS})_{\beta}$-sequence in $X(\Omega)$ for $J$ contains a convergent subsequence.

By Willem [14], for any $\beta \in \mathbb{R}$, a (PS) $\beta$-sequence in $X(\Omega)$ for $J$ is bounded. Moreover, a (PS)-value $\beta$ should be nonnegative.

Lemma 2.3. Let $\beta \in \mathbb{R}$ and $\left\{u_{n}\right\}$ be a $(P S)_{\beta}$-sequence in $X(\Omega)$ for $J$, then there exists a positive number $c(\beta)$ such that $\left\|u_{n}\right\|_{H^{1}} \leq c(\beta)$ for large $n$. Furthermore,

$$
a\left(u_{n}\right)=b\left(u_{n}\right)+o(1)=\frac{2 p}{p-2} \beta+o(1)
$$

and $\beta \geq 0$. Moreover, $c(\beta)$ can be chosen so that $c(\beta) \rightarrow 0$ as $\beta \rightarrow 0$.

Now, we consider the Nehari minimization problem

$$
\alpha_{X}(\Omega)=\inf _{u \in \mathbf{M}(\Omega)} J(u)
$$

where $\mathbf{M}(\Omega)=\{u \in X(\Omega) \backslash\{0\} \mid a(u)=b(u)\}$. Note that $\mathbf{M}(\Omega)$ contains every nonzero solution of (1.1) in $\Omega, \alpha_{X}(\Omega)>0$ and if $u_{0} \in \mathbf{M}(\Omega)$ achieves $\alpha_{X}(\Omega)$, then $u_{0}$ is a positive (or negative) solution of $(1.1)$ in $\Omega$ (see $[13,14]$ ). Moreover, we have the following useful lemma, whose proof can be found in [13, Lemma 7].

Lemma 2.4. Let $\left\{u_{n}\right\}$ be in $X(\Omega)$. Then, $\left\{u_{n}\right\}$ is a $(P S)_{\alpha_{X}(\Omega)}$-sequence in $X(\Omega)$ for $J$ if and only if $J\left(u_{n}\right)=\alpha_{X}(\Omega)+\mathrm{o}(1)$ and $a\left(u_{n}\right)=b\left(u_{n}\right)+\mathrm{o}(1)$.

We denote

(i) $\alpha_{X}(\Omega)$ by $\alpha_{0}(\Omega)$ for $X(\Omega)=H_{0}^{1}(\Omega)$ and $\alpha_{X}(\Omega)$ by $\alpha_{s}(\Omega)$ for $X(\Omega)=H_{s}(\Omega)$,

(ii) $\mathbf{M}(\Omega)$ by $\mathbf{M}_{0}(\Omega)$ for $X(\Omega)=H_{0}^{1}(\Omega)$ and $\mathbf{M}(\Omega)$ by $\mathbf{M}_{s}(\Omega)$ for $X(\Omega)=H_{s}(\Omega)$.

Remark 2.5. By the principle of symmetric criticality (see [11]), we have every $(\mathrm{PS})_{\beta}$ sequence in $X(\Omega)$ for $J$ is a $(\mathrm{PS})_{\beta}$-sequence in $H_{0}^{1}(\Omega)$ for $J$.

Let $\Omega$ be any unbounded domain and $\xi \in C^{\infty}([0, \infty))$ such that $0 \leq \xi \leq 1$ and

$$
\xi(t)= \begin{cases}0 & \text { for } t \in[0,1] \\ 1 & \text { for } t \in[2, \infty)\end{cases}
$$


Let

$$
\xi_{n}(z)=\xi\left(\frac{2|z|}{n}\right)
$$

Then, we have the following results whose proof can be found in [15].

Proposition 2.6. Equation (1.1) in $\Omega$ does not admit any solution $u_{0}$ such that $J\left(u_{0}\right)=$ $\alpha_{X}(\Omega)$ if and only if for each $(P S)_{\alpha_{X}(\Omega)}$-sequence $\left\{u_{n}\right\}$ in $X(\Omega)$ for $J$, there exists a subsequence $\left\{u_{n}\right\}$ such that $\left\{\xi_{n} u_{n}\right\}$ is also a $(P S)_{\alpha_{X}(\Omega)}$-sequence in $X(\Omega)$ for $J$.

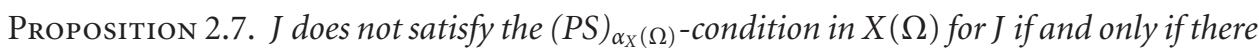
exists a $(P S)_{\alpha_{X}(\Omega)}$-sequence $\left\{u_{n}\right\}$ in $X(\Omega)$ for J such that $\left\{\xi_{n} u_{n}\right\}$ is also a $(P S)_{\alpha_{X}(\Omega)}$-sequence in $X(\Omega)$ for $J$.

Let $\Omega_{1} \varsubsetneqq \Omega_{2}$, clearly $\alpha_{X}\left(\Omega_{1}\right) \geq \alpha_{X}\left(\Omega_{2}\right)$. Then, we have the following useful results.

Lemma 2.8. Let $\Omega_{1} \varsubsetneqq \Omega_{2}$ and $J: X\left(\Omega_{2}\right) \rightarrow \mathbb{R}$ be the energy functional. Suppose that $\alpha_{X}\left(\Omega_{1}\right)$ $=\alpha_{X}\left(\Omega_{2}\right)$. Then, the following hold:

(i) equation (1.1) in $\Omega_{1}$ does not admit any solution $u_{0} \in X\left(\Omega_{1}\right)$ such that $J\left(u_{0}\right)=$ $\alpha_{X}\left(\Omega_{1}\right)$;

(ii) J does not satisfy the $(P S)_{\alpha_{X}\left(\Omega_{2}\right)}$-condition.

The proof is given by Wang and Wu [13, Lemma 13].

By the Rellich compact theorem, $J$ satisfies the (PS $)_{\alpha_{X}(\Omega)}$-condition in $X(\Omega)$ if $\Omega$ is a bounded domain.

Lemma 2.9. Let $\Omega$ be a bounded domain in $\mathbb{R}^{N}$. Then, the $(P S)_{\alpha_{X}(\Omega) \text {-condition holds in }}$ $X(\Omega)$ for J. Furthermore, (1.1) in $\Omega$ has a positive solution $u_{0}$ such that $J\left(u_{0}\right)=\alpha_{X}(\Omega)$.

\section{Concentration behavior}

We need the following results.

Lemma 3.1. Let $\Omega$ be an unbounded domain. Then,

$$
\alpha_{X}(\Omega(r)) \searrow \alpha_{X}(\Omega) \text { as } r \succ \infty .
$$

Proof. Since $\Omega(r)$ is a bounded domain for all $r>0$, by Lemmas 2.8 and 2.9, we have $\alpha_{X}(\Omega(r))$ is monotone decreasing as $r$ is monotone increasing and $\alpha_{X}(\Omega(r))>\alpha_{X}(\Omega)$. Thus, there exists a $d_{0} \geq \alpha_{X}(\Omega)$ such that

$$
\alpha_{X}(\Omega(r)) \succ d_{0} \quad \text { as } r \succ \infty .
$$

Claim that $d_{0} \leq \alpha_{X}(\Omega)$. Let $\left\{u_{n}\right\}$ be a (PS $\alpha_{X}(\Omega)$-sequence in $X(\Omega)$ for J. By Lemma 2.3, there exists a $c>0$ such that

$$
\int_{\Omega}\left|\nabla u_{n}\right|^{2}+u_{n}^{2} \leq c, \quad \int_{\Omega}\left|u_{n}\right|^{p} \leq c
$$




\section{Symmetry and concentration behavior}

for all $n \in \mathbb{N}$. Thus, for each $n \in \mathbb{N}$, there exists a sequence $\left\{r_{n}\right\}$ such that $r_{n}>0$ with $r_{n} \succ \infty$ as $n \rightarrow \infty$ and

$$
\int_{\Omega \cap\left\{|z| \geq r_{n}\right\}}\left|\nabla u_{n}\right|^{2}+u_{n}^{2}<\frac{1}{n}, \quad \int_{\Omega \cap\left\{|z| \geq r_{n}\right\}}\left|u_{n}\right|^{p}<\frac{1}{n} .
$$

Now, define $\eta_{r_{n}}(z)=\eta\left(2|z| / r_{n}\right)$, where $\eta \in C_{c}^{\infty}([0, \infty))$ satisfies $0 \leq \eta \leq 1$ and

$$
\eta(t)= \begin{cases}1 & \text { for } t \in[0,1] \\ 0 & \text { for } t \in[2, \infty)\end{cases}
$$

Then, $\eta_{r_{n}} u_{n} \in X(\Omega)$. From (3.4), we obtain

$$
\begin{aligned}
& a\left(\eta_{r_{n}} u_{n}\right)=a\left(u_{n}\right)+o(1), \\
& b\left(\eta_{r_{n}} u_{n}\right)=b\left(u_{n}\right)+o(1) .
\end{aligned}
$$

By the routine computations, there exists a sequence $\left\{s_{n}\right\} \subset \mathbb{R}^{+}$such that $a\left(s_{n} \eta_{r_{n}} u_{n}\right)=$ $b\left(s_{n} \eta_{r_{n}} u_{n}\right), s_{n}=1+o(1)$ and

$$
J\left(s_{n} \eta_{r_{n}} u_{n}\right)=J\left(\eta_{r_{n}} u_{n}\right)+o(1)=\alpha_{X}(\Omega)+o(1),
$$

that is, $s_{n} \eta_{r_{n}} u_{n} \in \mathbf{M}\left(\Omega\left(r_{n}\right)\right)$ and $J\left(s_{n} \eta_{r_{n}} u_{n}\right) \geq \alpha_{X}\left(\Omega\left(r_{n}\right)\right)=d_{0}+o(1)$. Taking $n \rightarrow \infty$, we get $\alpha_{X}(\Omega) \geq d_{0}$. Therefore, $\alpha_{X}(\Omega)=d_{0}$.

Let $\Omega_{t}^{+}=\{(x, y) \in \Omega \mid y>t\}$ and $\Omega_{t}^{-}=\{(x, y) \in \Omega \mid y<t\}$. Then, we have the following result.

Lemma 3.2. Suppose that the domain $\Omega$ satisfies $(\Omega 1),(\Omega 2)$, and $(\Omega 3)$. Then, for each $\varepsilon>0$ and $l \geq 0$, there exists a $\delta(\varepsilon, l)>0$ such that if $u \in \mathbf{M}_{0}(\Omega)$ and $J(u)<\alpha_{0}(\Omega)+\delta(\varepsilon, l)$, then either $\int_{\Omega_{l}^{+}}|u|^{p}<\varepsilon$ or $\int_{\Omega_{-l}^{-}}|u|^{p}<\varepsilon$.

Proof. If not, there exist $c>0, l_{0} \geq 0$, and $\left\{u_{n}\right\} \subset \mathbf{M}_{0}(\Omega)$ such that $J\left(u_{n}\right)=\alpha_{0}(\Omega)+o(1)$,

$$
\int_{\Omega_{l_{0}}^{+}}\left|u_{n}\right|^{p} \geq c, \quad \int_{\Omega_{-l_{0}}^{-}}\left|u_{n}\right|^{p} \geq c
$$

By Lemma 2.4, $\left\{u_{n}\right\}$ is a $(\mathrm{PS})_{\alpha_{0}(\Omega)}$-sequence in $H_{0}^{1}(\Omega)$ for $J$. Now, $\Omega$ satisfies condition $(\Omega 3)$. By Proposition 2.6, there exists a subsequence $\left\{u_{n}\right\}$ such that $\left\{\xi_{n} u_{n}\right\}$ is also 


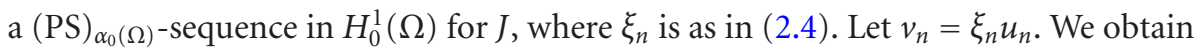

$$
\begin{aligned}
J\left(v_{n}\right) & =\alpha_{0}(\Omega)+\mathrm{o}(1), \\
J^{\prime}\left(v_{n}\right) & =\mathrm{o}(1) \quad \text { in } H^{-1}(\Omega) .
\end{aligned}
$$

Since $\Omega$ is a $y$-symmetric domain in $\mathbb{R}^{N}$ separated by a bounded domain, there exists a $n_{0}>l_{0}$ such that $v_{n}=0$ in $\overline{\Omega\left(n_{0}\right)}$ for $n>2 n_{0}$, and $\Omega \backslash \overline{\Omega\left(n_{0}\right)}=\Omega_{1} \cup \Omega_{2}$, where $\Omega_{1}=\Omega_{n_{0}}^{+}$ and $\Omega_{2}=\Omega_{-n_{0}}^{-}$. Moreover, $v_{n}=v_{n}^{1}+v_{n}^{2}$, where

$$
v_{n}^{i}(z)=\left\{\begin{array}{ll}
v_{n}(z) & \text { for } z \in \Omega_{i} \\
0 & \text { for } z \notin \Omega_{i}
\end{array} \quad \text { for } i=1,2 .\right.
$$

Then, $v_{n}^{i} \in H_{0}^{1}\left(\Omega_{i}\right)$ and $a\left(v_{n}^{i}\right)=b\left(v_{n}^{i}\right)+o(1)$. By (3.9), we obtain

$$
J^{\prime}\left(v_{n}^{i}\right)=\mathrm{o}(1) \text { strongly in } H^{-1}\left(\Omega_{i}\right) \quad \text { for } i=1,2 .
$$

Assume that

$$
J\left(v_{n}^{i}\right)=c_{i}+\mathrm{o}(1) \quad \text { for } i=1,2 .
$$

Since $J\left(v_{n}\right)=J\left(v_{n}^{1}\right)+J\left(v_{n}^{2}\right)=\alpha_{0}(\Omega)+o(1)$, we have $c_{1}+c_{2}=\alpha_{0}(\Omega)$. Since $c_{i}$ are (PS)values in $H_{0}^{1}\left(\Omega_{i}\right)$ for $J$, by Lemma $2.3, c_{i} \geq 0$ and

$$
\begin{aligned}
& c_{1}\left(\frac{2 p}{p-2}\right)=\int_{\Omega_{l_{0}}^{+}}\left|v_{n}^{1}\right|^{p}+o(1)=\int_{\Omega_{l_{0}}^{+}}\left|u_{n}\right|^{p}+o(1), \\
& c_{2}\left(\frac{2 p}{p-2}\right)=\int_{\Omega_{-l_{0}}^{-}}\left|v_{n}^{2}\right|^{p}+o(1)=\int_{\Omega_{-l_{0}}^{-}}\left|u_{n}\right|^{p}+o(1) .
\end{aligned}
$$

By (3.8), we have $c_{i}>0$ for $i=1,2$. We have that

$$
\alpha_{0}(\Omega)=c_{1}+c_{2} \geq \alpha_{0}\left(\Omega_{1}\right)+\alpha_{0}\left(\Omega_{2}\right)
$$

which contradicts the fact that $\alpha_{0}(\Omega) \leq \alpha_{0}\left(\Omega_{i}\right)$ for $i=1,2$.

Now, we begin to show the proof of Theorem 1.1. By Lemma 3.1, for each $\varepsilon>0$ and $l \geq 0$, there exists a $\delta(\varepsilon, l)>0$ such that if $u \in \mathbf{M}_{0}(\Omega)$ and $J(u)<\alpha_{0}(\Omega)+\delta(\varepsilon, l)$, then $\int_{\Omega_{l}^{+}}|u|^{p}<\varepsilon$ or $\int_{\Omega_{-l}^{-}}|u|^{p}<\varepsilon$. Moreover, by Lemma 3.2, there exists an $\tilde{r}>0$ such that 
$\alpha_{0}(\Omega(r))<\alpha_{0}(\Omega)+\delta(\varepsilon)$ for all $r>\tilde{r}$. Thus, if $v$ is a ground-state solution of (1.1) in $H_{0}^{1}(\Omega(r))$ for $r>\tilde{r}$, then $v \in \mathbf{M}_{0}(\Omega(r)) \subset \mathbf{M}_{0}(\Omega), J(v)<\alpha_{0}(\Omega)+\delta(\varepsilon)$ and either $\int_{\Omega_{l}^{+}}|v|^{p}<\varepsilon$ or $\int_{\Omega_{-l}^{-}}|v|^{p}<\varepsilon$.

Now, we begin to show the proof of Theorem 1.3.

(i) By Lemma 3.1, we have $J\left(v_{m}\right)=\alpha_{0}(\Omega(m))=\alpha_{0}(\Omega)+o(1)$. Since $v_{m} \in \mathbf{M}_{0}(\Omega(m)) \subset$

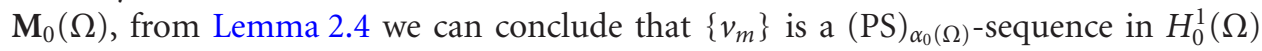
for $J$.

(ii) Let $v \in L^{q}(\Omega)$, where $1 / p+1 / q=1$. Then, for each $\varepsilon>0$ there exists an $l>0$ such that

$$
\int_{(\Omega(l))^{c}}|v|^{q}<\varepsilon^{q}
$$

By Theorem 1.1, there exists an $m_{0}>l$ such that

$$
\int_{\Omega(l)}\left|v_{m}\right|^{q}<\varepsilon^{p} \quad \forall m>m_{0}
$$

Thus, for each $\varepsilon>0$ there exists an $m_{0}$ such that

$$
\begin{aligned}
\int_{\Omega} v_{m} v= & \int_{(\Omega(l))^{c}} v_{m} v+\int_{\Omega(l)} v_{m} v \leq\left(\int_{(\Omega(l))^{c}}\left|v_{m}\right|^{p}\right)^{1 / p}\left(\int_{(\Omega(l))^{c}}|v|^{q}\right)^{1 / q} \\
& +\left(\int_{\Omega(l)}\left|v_{m}\right|^{p}\right)^{1 / p}\left(\int_{\Omega(l)}|v|^{q}\right)^{1 / q} \leq\left(c_{1}+c_{2}\right) \varepsilon \quad \forall m>m_{0}
\end{aligned}
$$

where $c_{1}=\left((2 p /(p-2)) \alpha_{0}(\Omega)\right)$ and $c_{2}=\|v\|_{L^{q}}$. This implies that $v_{m} \rightarrow 0$ weakly in $L^{p}(\Omega)$ as $m \rightarrow \infty$. Since $v_{m}$ is a solution of $(1.1)$ in $\Omega(m)$, we have

$$
\int_{\Omega(m)} \nabla v_{m} \nabla \varphi+v_{m} \varphi=\int_{\Omega(m)}\left|v_{m}\right|^{p-2} v_{m} \varphi \quad \forall \varphi \in H_{0}^{1}(\Omega(m))
$$

First, we need to show for each $\varepsilon>0$ and $\varphi \in C_{c}^{1}(\mathbf{S})$ there exists an $m_{0}$ such that

$$
\int_{\Omega(m)} \nabla v_{m} \nabla \varphi+v_{m} \varphi<\varepsilon \quad \forall m>m_{0}
$$

for $\varphi \in C_{c}^{1}(\Omega)$. Let $K=\operatorname{supp} \varphi$, then $K \subset \Omega$ is compact and there exists an $m_{1}$ such that $K \subset \Omega(m)$ for all $m \geq m_{1}$. From Theorem 1.4, for each $\varepsilon>0$ there exist $l_{0}>0$ and $m_{0}$ such that $\varphi \in H_{0}^{1}(\Omega(m))$,

$$
\int_{\left(\Omega\left(l_{0}\right)\right)^{c}}|\varphi|^{p}=0, \quad \int_{\Omega\left(l_{0}\right)}\left|v_{m}\right|^{p}<\varepsilon^{(p-1) / p} \quad \forall m>m_{0} .
$$


We obtain

$$
\begin{aligned}
\int_{\Omega(m)}\left|v_{m}\right|^{p-2} v_{m} \varphi & =\int_{\left(\Omega\left(l_{0}\right) c^{c}\right.}\left|v_{m}\right|^{p-2} v_{m} \varphi+\int_{\Omega\left(l_{0}\right)}\left|v_{m}\right|^{p-2} u_{m}^{1} \varphi \\
\leq & \left(\int_{\left(\Omega\left(l_{0}\right) c\right.}\left|v_{m}\right|^{p}\right)^{(p-1) / p}\left(\int_{\left(\Omega\left(l_{0}\right)\right)^{c}}|\varphi|^{p}\right)^{1 / p} \\
& +\left(\int_{\Omega\left(l_{0}\right)}\left|v_{m}\right|^{p}\right)^{(p-1) / p}\left(\int_{\Omega\left(l_{0}\right)}|\varphi|^{p}\right)^{1 / p} \\
\leq & c \mathcal{E}, \\
\int_{\Omega} \nabla v_{m} \nabla \varphi+\int_{\Omega} v_{m} \varphi= & \int_{\Omega(m)} \nabla v_{m} \nabla \varphi+\int_{\Omega(m)} v_{m} \varphi \\
= & \int_{\Omega(m)}\left|v_{m}\right|^{p-2} v_{m} \varphi \quad \forall m>m_{0} .
\end{aligned}
$$

We have that

$$
\int_{\Omega} \nabla v_{m} \nabla \varphi+\int_{\Omega} v_{m} \varphi \leq c \mathcal{E} \quad \forall m>m_{0}
$$

Since $\alpha_{0}(\Omega(m+1))<\alpha_{0}(\Omega)$, there exists a $C>0$ such that $\left\|v_{m}\right\|_{H^{1}} \leq C$. Thus, for each $\varepsilon>0$ and $\psi \in H_{0}^{1}(\Omega)$, there exists a $\varphi \in C_{c}^{1}(\Omega)$ such that

$$
\|\psi-\varphi\|_{H^{1}}<\frac{\varepsilon}{C}
$$

From (3.23) and (3.24), we can conclude that for each $\varepsilon>0$ and $\psi \in H_{0}^{1}(\Omega)$ there exists an $m_{0}>0$ such that

$$
\begin{aligned}
\left\langle v_{m}, \psi\right\rangle_{H^{1}} & =\left\langle v_{m}, \psi-\varphi\right\rangle_{H^{1}}+\left\langle v_{m}, \varphi\right\rangle_{H^{1}} \\
& \leq C\|\psi-\varphi\|_{H^{1}}+\left\langle v_{m}, \varphi\right\rangle_{H^{1}} \\
& <\varepsilon+c \varepsilon \text { for } m>m_{0} .
\end{aligned}
$$

This implies that $v_{m} \rightarrow 0$ weakly in $H_{0}^{1}(\Omega)$.

\section{Symmetry}

Now, we begin to show the proof of Theorem 1.4. Let $v$ be a ground-state solution of (1.7) in $\Theta$ and let $z^{*}=(x,-y)$ be the reflection point of $z=(x, y)$ with respect to the hyperplane $T:=\{y=0\}$. First, we claim that either

$$
v(z) \geq v\left(z^{*}\right) \quad \forall z \in \Theta^{+}
$$

or

$$
v(z) \leq v\left(z^{*}\right) \quad \forall z \in \Theta^{+},
$$


1028 Symmetry and concentration behavior

where $\Theta^{+}$is one of half domain $\Theta \backslash T$. If not, then the following two sets

$$
\begin{aligned}
& A_{+}=\left\{z \in \Theta^{+} \mid v(z)>v\left(z^{*}\right)\right\} \\
& A_{-}=\left\{z \in \Theta^{+} \mid v(z)<v\left(z^{*}\right)\right\}
\end{aligned}
$$

are nonempty. Let $w(z)=v(z)-v\left(z^{*}\right)$ for $z \in \Theta^{+}$. Then, $w$ satisfies

$$
\begin{gathered}
\Delta w-w+f_{v}(\zeta(z)) w=0, \quad \text { in } \Theta^{+}, \\
w=0, \quad \text { in } \partial \Theta^{+},
\end{gathered}
$$

where $\zeta(z)$ is between $v(z)$ and $v\left(z^{*}\right)$. Let

$$
A_{-}^{*}=\left\{z^{*} \mid z \in A_{-}\right\} .
$$

For $d>0$, we define a function

$$
u_{d}(z)= \begin{cases}w(z) & \text { if } z \in A_{+} \\ d w\left(z^{*}\right) & \text { if } z \in A_{-}^{*} \\ 0 & \text { otherwise }\end{cases}
$$

Since $\int_{A_{+}} w \phi_{1}>0$ and $\int_{A_{-}} w \phi_{1}<0$, there exists a constant $d_{0}>0$ such that

$$
\int_{\Theta} u_{d_{0}} \phi_{1}=\int_{A_{+}} w \phi_{1}+d_{0} \int_{A_{-}} w \phi_{1}=0,
$$

where $\phi_{1}$ is the first positive eigenfunction of the following eigenvalue problem:

$$
\begin{gathered}
\left(\Delta-1+f_{v}(\zeta(z))\right) \phi+\lambda \phi=0 \quad \text { in } \Theta, \\
\phi=0 \text { on } \partial \Theta .
\end{gathered}
$$

Let $\lambda_{2}$ be the second eigenvalue of (4.9). Since $v$ is a ground-state solution of (1.7), by the same method of the proof of Theorem 2.11 in [10], we have $\lambda_{2}$ is nonnegative. Moreover, by (4.3)-(4.7), we have

$$
\begin{array}{lc}
\Delta u_{d}-u_{d}+f_{v}(\zeta(z)) u_{d}>0 & \text { for } z \in A_{+}, \\
\Delta u_{d}-u_{d}+f_{v}(\zeta(z)) u_{d}<0 & \text { for } z \in A_{-}^{*}, \\
\Delta u_{d}-u_{d}+f_{v}(\zeta(z)) u_{d}=0 & \text { otherwise. }
\end{array}
$$


Therefore, from (4.8) and (4.10), we have

$$
\begin{aligned}
0 & >\int_{\Theta}-u_{d}(z)\left[\Delta u_{d}(z)-u_{d}+f_{v}(\zeta(z)) u_{d}(z)\right] d z \\
& =\int_{\Theta}\left[\left|\nabla u_{d}(z)\right|^{2}+u_{d}^{2}-f_{v}(\zeta(z)) u_{d}^{2}(z)\right] d z \\
& \geq \lambda_{2} \int_{\Theta} u_{d}^{2}(z) d z \geq 0,
\end{aligned}
$$

a contradiction. This proves inequalities (4.1) and (4.2). By (4.1) and (4.2), we may assume $w(z) \geq 0$ for all $z \in \Theta^{+}$, if $w(z)>0$ for some $z \in \Theta^{+}$. Since $w$ satisfies (4.5), by using the strong maximum principle, we have $w>0$ in $\Theta^{+}$. Similarly, if $w(z) \leq 0$ and $w(z)<0$ for some $z \in \Theta^{+}$, we have $w<0$ in $\Theta^{+}$. Suppose that $w(z)>0$ for all $z \in \Theta^{+}$. Then, from (4.5) and applying the Hopf Lemma, we have

$$
\frac{\partial w}{\partial(-y)}\left(z_{0}\right)=-2 \frac{\partial v}{\partial y}\left(z_{0}\right)<0
$$

Similarly, if $w(z)<0$ for all $z \in \Theta^{+}$, we have $(\partial v / \partial) y\left(z_{0}\right)<0$, this contradicts the fact that $(\partial v / \partial) y\left(z_{0}\right)=0$. Therefore, $w(z)=0$ for all $z \in \Theta^{+}$or $v(x, y)=v(x,-y)$ for all $(x, y) \in \Theta$. The converse is obvious.

\section{Acknowledgment}

The author is partially supported by the National Science Council of Taiwan.

\section{References}

[1] A. Ambrosetti and P. H. Rabinowitz, Dual variational methods in critical point theory and applications, J. Functional Analysis 14 (1973), 349-381.

[2] J. Byeon, Existence of large positive solutions of some nonlinear elliptic equations on singularly perturbed domains, Comm. Partial Differential Equations 22 (1997), no. 9-10, 1731-1769.

[3] Nonlinear elliptic problems on singularly perturbed domains, Proc. Roy. Soc. Edinburgh Sect. A 131 (2001), no. 5, 1023-1037.

[4] J.-L. Chern and C.-S. Lin, The symmetry of least-energy solutions for semilinear elliptic equations, J. Differential Equations 187 (2003), no. 2, 240-268.

[5] E. N. Dancer, The effect of domain shape on the number of positive solutions of certain nonlinear equations, J. Differential Equations 74 (1988), no. 1, 120-156.

[6] B. Gidas, W. M. Ni, and L. Nirenberg, Symmetry and related properties via the maximum principle, Comm. Math. Phys. 68 (1979), no. 3, 209-243.

[7] Q. Han and F. Lin, Elliptic Partial Differential Equations, Courant Lecture Notes in Mathematics, vol. 1, Courant Institute of Mathematical Sciences, New York University, New York, 1997.

[8] S. Jimbo, Singular perturbation of domains and the semilinear elliptic equation. II, J. Differential Equations 75 (1988), no. 2, 264-289.

[9] W. C. Lien, S. Y. Tzeng, and H. C. Wang, Existence of solutions of semilinear elliptic problems on unbounded domains, Differential Integral Equations 6 (1993), no. 6, 1281-1298.

[10] C. S. Lin and W.-M. Ni, On the diffusion coefficient of a semilinear Neumann problem, Calculus of Variations and Partial Differential Equations (Trento, 1986), Lecture Notes in Math., vol. 1340, Springer, Berlin, 1988, pp. 160-174. 


\section{Symmetry and concentration behavior}

[11] R. S. Palais, The principle of symmetric criticality, Comm. Math. Phys. 69 (1979), no. 1, 19-30.

[12] H.-C. Wang, A Palais-Smale approach to problems in Esteban-Lions domains with holes, Trans. Amer. Math. Soc. 352 (2000), no. 9, 4237-4256.

[13] H.-C. Wang and T. F. Wu, Symmetry breaking in a bounded symmetry domain, NoDEANonlinear Differential Equations Appl. (2004), no. 11, 361-377.

[14] M. Willem, Minimax Theorems, Progress in Nonlinear Differential Equations and their Applications, Birkhäuser Boston Inc., Massachusetts, 1996.

[15] T.-F. Wu, Concentration and dynamic system of solutions for semilinear elliptic equations, Electron. J. Differential Equations (2003), no. 81, 1-14.

Tsung-Fang Wu: Center for General Education, Southern Taiwan University of Technology, Tainan 71005, Taiwan

E-mail address: tfwu@mail.stut.edu.tw 


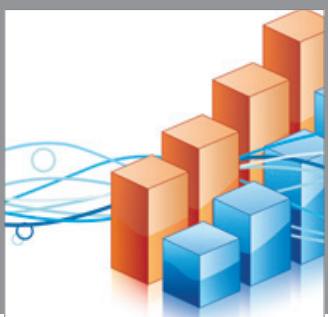

Advances in

Operations Research

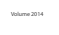

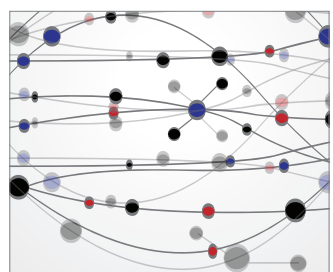

\section{The Scientific} World Journal
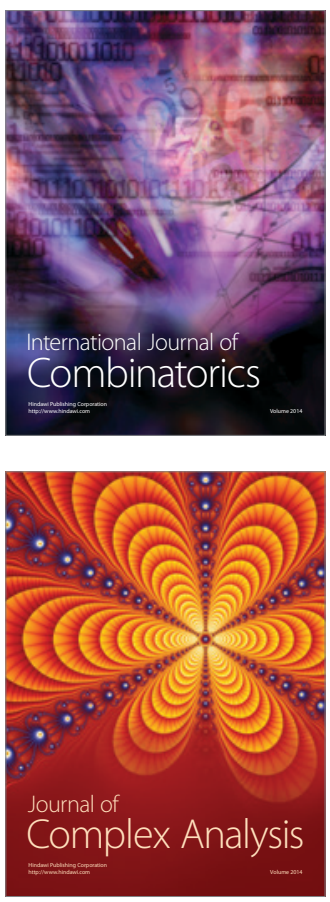

International Journal of

Mathematics and

Mathematical

Sciences
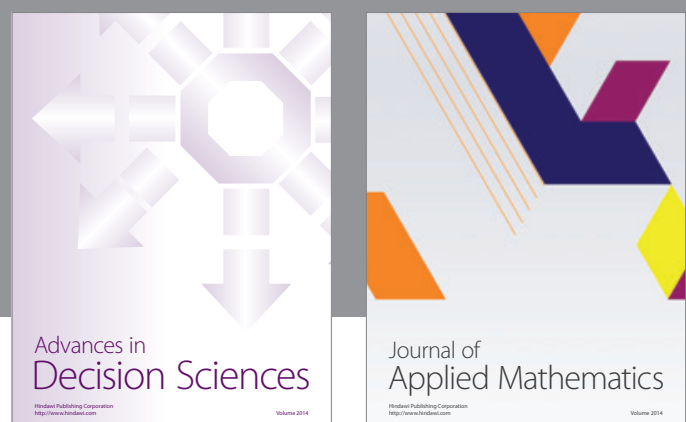

Journal of

Applied Mathematics
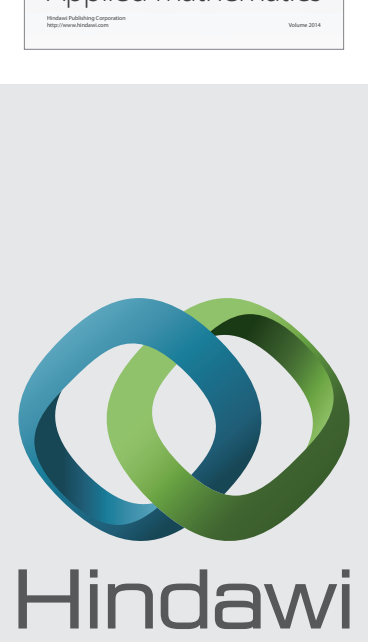

Submit your manuscripts at http://www.hindawi.com
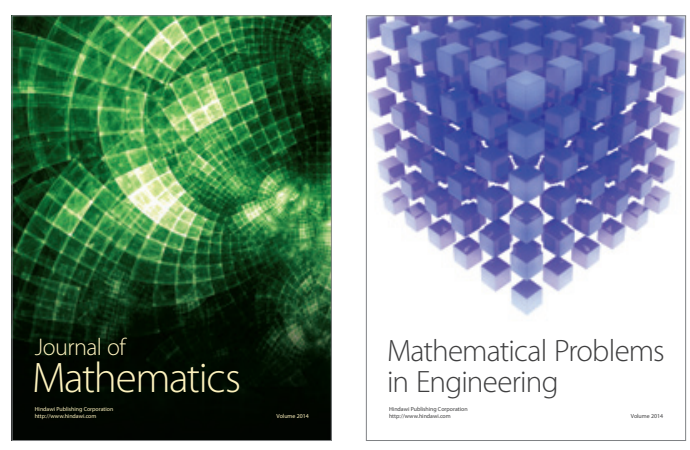

Mathematical Problems in Engineering
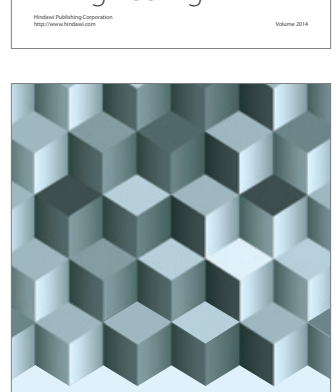

Journal of

Function Spaces
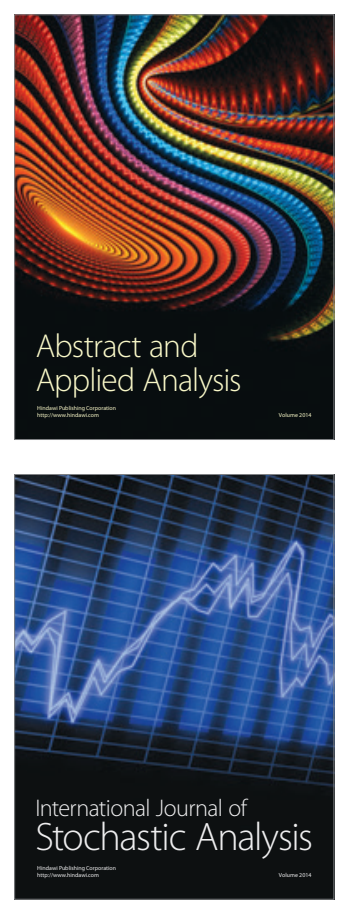

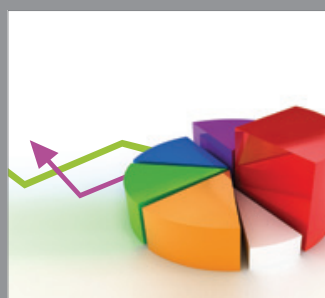

ournal of

Probability and Statistics

Promensencen
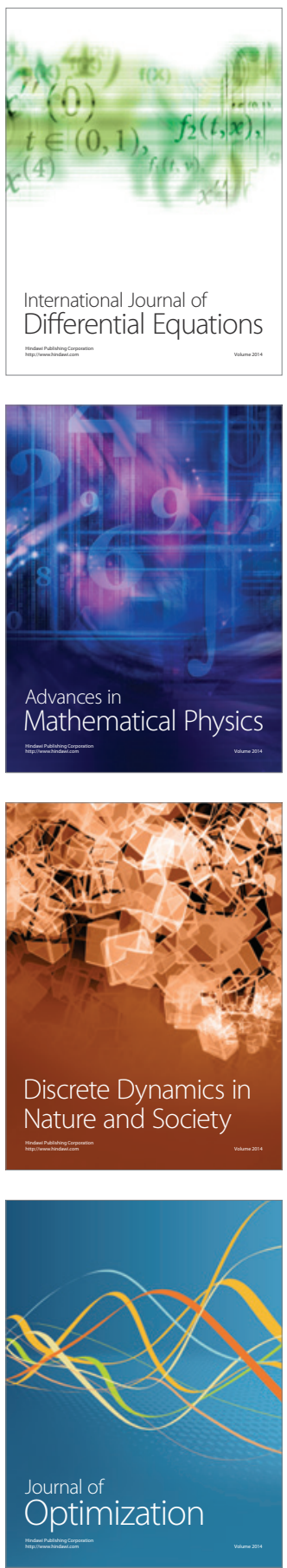